by a change in operation the second unit could deal with the whole of the output of the first unit.-and this has been effected.

In Imperial Chemical Industries, Ltd., four examples testify to the improvements which have been made by the introduction of work study. Thus, a drum washing plant team was reduced from five to three men and the total output increased by 50 per cent, representing a saving of $£ 1,200$ a year.

A procedure for bagging powder had been operated for several years, during which time there had been minor changes in the requirements of sieving and blending, and the material of the bags had changed. No obvious improvement of the long-established method appeared possible, but careful study of method indicated the possibility for a better flow of materials. As a result, the distance the powder was moved was reduced by almost 60 per cent and the distance the operator moved for each bag of powder was reduced by nearly 50 per cent. With no change in the level of production, it was possible to reduce from two operators to one. In the third example, cast-iron cooling-water pipes have to be tested under pressure and checked for straightness. By the original method used, two men could test 180 pipes in a day. Method study showed various possible improvements, and the one selected involved changes in equipment which cost only $£ 25$. One man can now test two hundred pipes in a day, and no incentives are involved in this case.

In another works, the inspection and servicing of cars and lorries was changed to a carefully worked out basis of incentive. As a result, the four shift operatives increased their output by 48 per cent and earned 26 per cent more, with a reduction in labour cost per unit of output of more than 15 per cent.

\title{
IMMUNOLOGICAL DISCRIMINATION BETWEEN THE BLOOD OF NORMAL AND OF TUMOUR-BEARING RATS
}

\author{
By DR. D. A. DARCY \\ Chester Beatty Research Institute (Institute of Cancer Research: Royal Cancer Hospital), London, S.W.3
}

$\mathrm{U}$ SING the gel diffusion technique of Ouchterlony ${ }^{1}$, it has been found possible to distinguish the blood plasma (or serum) of rats bearing transplanted or induced tumours from the plasma of normal rats or rats bearing homografts of normal tissues.

The method was, first, to prepare antisera of high precipitin titre in rabbits by injecting them with the plasma or serum of $(a)$ normal rats or $(b)$ rats bearing the transplanted Walker tumour. Other rabbits were inoculated with the cells of the Walker tumour; but the antisera so obtained had only very weak discriminatory power, no more than would be expected from the incidental contamination of the inoculated cells with plasma. When antisera of satisfactory strength had been obtained, they were allowed to diffuse through an agar gel to meet and react with the antigens (rat plasma) diffusing in the opposite direction. In this way a series of bands or lines of precipitate was formed in the gel giving a 'spectrum' characteristic of the antigenic structure of the plasma in question, the antigen structure being determined by the proteins, and possibly other soluble substances. It was found that the precipitin spectrum of the cancer plasma differed from the normal in a charac. teristic way.

A variety of transplantable rat tumours was employed, namely, the Walker tumour, the Yoshida sarcoma, the $R 48$ sarcoma, the $G D-1$ tumour, the August tumour and a sarcoma induced by means of xanthine. The induced tumours were a series of sarcomas of various sizes arising at the site of implanted pellets of benzpyrene, and one hepatoma induced by feeding butter yellow. The rats used throughout both for controls and for tumour-bearing were from a colony of non-inbred white rats originally derived from the Wistar strain, the only exceptions being that the August tumour and the Yoshida tumour were grown in the August and $B D-1$ strains, respectively. The control plasmas came from rats which were either untreated or which bore homografts of normal tissue (liver and skin) at various stages of evolution.
Agar diffusion plates were prepared with three wells as shown in Figs. 1 and 2. The bottom well was charged with antiserum and the left and right wells with cancer and normal plasma, respectively. The lines of precipitate are composed of the various antigenic components in combination with their corresponding antibodies. When a line on one side fuses with one on the other side to form an arc, the antigenic components in question are identical ${ }^{1}$. When an are is displaced to one side, this indicates a lower concentration of the antigen on that side. When a line crosses to the opposite side without fusing or without deviating, the substance in question is either absent or in negligibly low concentration on that side.

The antiserum prepared against normal rat plasma (A.N.P.) was used in a large number of plate tests to compare the different cancer plasmas with the plasmas of the normal or grafted controls. Both pooled and single control plasmas were tested. It was found that the cancer plasmas differed from the controls in having a group of rather faint lines (labelled $K$ in Fig. 1) advancing towards the antiserum in front of the strong line which is the leading line $(A$, Fig. 1$)$ of the normal spectrum. The $K$ group was composed of two lines at first, but later others which may be 'immunological artifacts'" appeared among them. The $K$ lines were shown most strongly by the blood of animals bearing advanced tumours; but the antigens were clearly demonstrable in the blood as early as six days after implantation of the Walker tumour. The lines appeared to fuse with an unidentified group in the normal spectrum; the simple interpretation of them would be that they represent substances which are in higher concentration in the cancer blood than in the normal. However, it was found that a similar group of $K$ lines could be produced by autolysed whole normal rat blood, and occasionally by normal rat plasma that had been stored for a very long time. It is suggested, therefore, that the $K$ lines may represent partially broken-down substances (probably protein) which can diffuse faster than the parent substances 


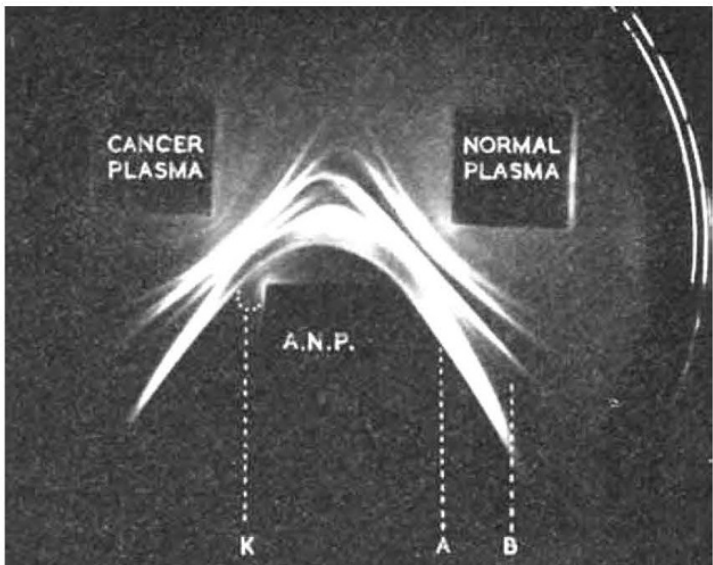

Fig. 1. A plate after nine days diffusion at room temperature. The cancer plasma comes from a rat bearing a 13-day old Walker tumour. The normal plasma was a pooled mixture from untreated controls, Both plasmas were diluted one part in ten with citrated saline. The antiserum (A.N.P.) had been prepared against normal rat plasma

while retaining their original antigenicity. Atypical single $K$ lines were also occasionally found in the plasmas of control rats described as 'bandaged and fod' (see below). A second difference observed was that the line $A$ was actually farther from the antibody well in the cancer plasma than in the normal, indicating a lower concentration of the antigen in question in the cancer blood. Certain other antigens in the cancer plasma appeared to be in lower concentration, often to a striking degree (for example, line $B$, Fig. 1) than in the normal. The reverse was also found, namely, an antigen or antigens in the cancer spectrum much stronger than in the control.

When the antiserum employed in the gel plates was that prepared against the plasma of animals bearing the Walker tumour, a more striking distinction was obtained. In addition to a $K$ group and a weaker $A$ antigen, the cancer plasmas now showed a line which was absent entirely from the pooled normal plasma (Fig. 2). If the antiserum was then absorbed to exhaustion with normal plasma, it still reacted with the cancer plasma either in the 'ring' test in glass tubes, or in the gel plates to give a band

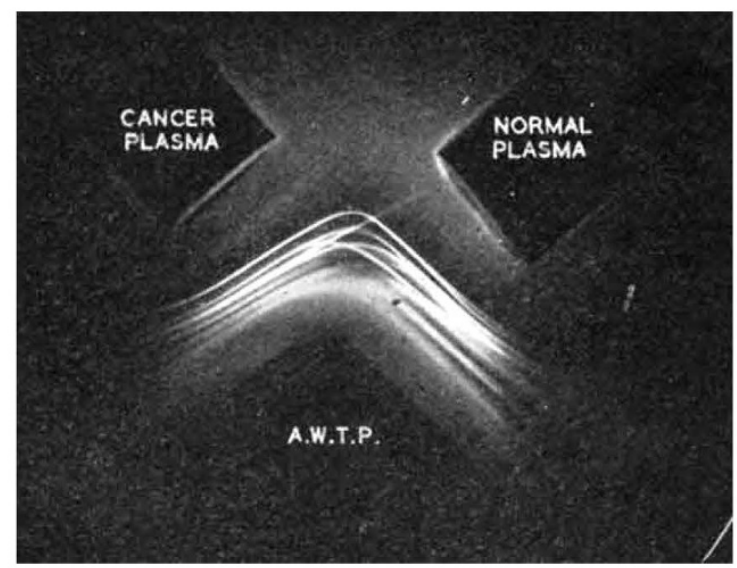

Fig. 2. A plate after ten days at room temperature. The cancer plasma was from a rat bearing a 12-day old Walker tumour. The normal plasma was a pooled mixture from untreated controls. Both plasmas were diluted one part in firteen with citrated saline. of animals bearing Walker tumours of precipitate, The blood plasmas of all animals bearing well-established tumours, including most sixday old Walker tumours, were found to give strongly positive ring tests with the absorbed antiserum, whereas the great majority of controls were negative. Blood plasmas of other species (man, mouse, hamster) bearing induced or spontaneous tumours were also negative.

A small percentage of control plasmas, all coming from a group of rats which had received skin homografts, however, was positive. Further investigation of this point showed that the skin grafting itself was not responsible, but that the effect was caused by the tight plaster bandage on chest and upper abdomen (such as is commonly used for skin grafting in rats and mice $^{3}$ ) acting in conjunction with insufficient fasting of the rats before bleeding. The bandage alone had no effect, and complete lack of fasting produced, at best, faint or doubtful positives. The two acting together produced strong positives. This antigen, therefore, cannot be regarded as cancerspecific; but it is interesting that it should be absent from blood of normal fasting rats. It was present in the blood of tumour-bearing animals even when they were starved for a day before bleeding, and even when the tumour was inoculated in the hind leg so as to avoid any possible 'corset' effect such as might occur when the tumour was grown in the usual way in the animal's side.

From this ovidence it seems likely that the antigen in question is a lipid or lipoprotein. Lipids are found in relatively high concentration in the blood of tumour-bearing rats. ${ }^{4}$. It was an easy matter to distinguish the plasma of the rats which had been bandaged and fed before bleeding from true cancer plasmas by means of the characteristic $K$ antigens and the weaker $A$ antigen of the latter.

None of the above antigen-lines has been identified chemically. However, special methods are becoming available for this purpose $e^{5}$, so that a complete characterization of the precipitin spectrum should eventually be possible. It may be speculated, for example, that the line labelled $A$ in the spectrum represents serum albumin; this protein has a smaller molecule than the globulins and would be expected to diffuse ahead of them ; it is, furthermore, reported to occur in lower concentration in cancer blood than in normal 6 .

In conclusion, it may be said that while the present results represent only a preliminary survey, the determination of the precipitin 'spectrum' of blood and other body fluids offers interesting possibilities clinically for diagnostic and other purposes, and experimentally in a wide variety of fields.

This investigation has been supported by grants to the Chester Beatty Research Institute (Institute of Cancer Research: Royal Cancer Hospital) from the British Empire Cancer Campaign, the Jane Coffin Childs Mernorial Fund for Medical Research, the Anna Fuller Fund and the National Cancer Institute of the National Institutes of Health, U.S. Public Health Service.

[July 11

${ }^{1}$ Ouchterlony, O., Ark. Kemi. Min. Geol., 26B, 1 (1948). Wilson, M. W., and Pringle , B. I., J. Immunol, 78, 232 (1954). Oudin J., "Methods in Medical Research", 5 (The Year Book Publishers, Chicago, 1951). Jennings, R. K.., J. Hact., 67, 659 (1954).

'Burtin, P., Bull. Ste. Chim. Biol., 38, 1021 (1954).

" Btllingham, R. E., and Medawar, P. B., J. Exp. Biol., 28,385 (1951). - Frederick, G. I., and Begg, R. W., Proc. Amer. Assoc. Cancer Res. 1. 14 (1954).

s Grabar, P., and Williams, J. A., Biachim. Biophys. Acta, 10, 193

1953). Bỉorklund, B., Proc. Soc. Exp. Biol. and Med., 85, 43

- Hauschka, T. \$., Cancer Research, 12, 615 (1952). 